\title{
Market Based Approaches for Dynamic Spectrum Assignment
}

\author{
Martin B.H. Weiss
}

\author{
William H. Lehr
}

\begin{abstract}
Much of the technical literature on spectrum sharing has been on developing technologies and systems for (non-cooperative) opportunistic use. In this paper, we situate this approach to secondary spectrum use in a broader context, one that includes cooperative approaches to Dynamic Spectrum Access (DSA). In this paper, we introduce readers to this broader approach to DSA by contrasting it with non-cooperative sharing (opportunistic use), surveying relevant literature, and suggesting future directions for research
\end{abstract}

Index Terms - Cognitive radio, Economics, Radio spectrum managements, Spectrum markets,

\section{INTRODUCTION}

$S_{\text {ine }}$ ince the emergence of wireless communications in the 1920s, governments have regulated the allocation and assignment of spectrum because it was seen as a scarce public resource. ${ }^{1}$ Demand for spectrum access rights exceeds the available supply. At the same time, measurements of spectrum suggest that, in most bands, spectrum is being underutilized [1]. Although the quantity of radio frequency spectrum is fixed, the available supply could be significantly expanded by dynamically sharing the spectrum more intensively.

Technologies that enable more dynamic spectrum sharing (e.g., smart antennas, software defined radios, new modulation schemes, etc.) may be utilized by service providers to increase the spectral efficiency of their licensed spectrum use. ${ }^{2}$ While

Manuscript received December 1, 2009.

Martin B.H. Weiss is with the Telecommunications Program, School of Information Sciences, University of Pittsburgh, Pittsburgh PA 15260 (email: mbw@pitt.edu). Willam H. Lehr is a research associate at the Laboratory for Computer Science at Massachusetts Institute of Technology

\footnotetext{
${ }^{1}$ We distinguish between spectrum allocation and spectrum assignment. Spectrum allocation is the process of associating an application with a frequency band (e.g., FM radio to $88-108 \mathrm{MHz}$ ). Spectrum assignment is the process of granting a specific usage right to a specific user in a specific location (e.g., $91.3 \mathrm{MHz}$ to Pittsburgh Community Broadcasting Corporation to operate an FM radio station in Pittsburgh at $18 \mathrm{KWatts}$ ). Allocation decisions are governed by a set of non-technical parameters (such as international agreements and social goals) as well as technical ones. Assignment decisions are governed by political, economic and institutional factors. As a result, it is useful to separate these types of decisions. This paper will be focussed on spectrum assignment.

${ }^{2}$ For example, the migration to $4 \mathrm{G}$ LTE from $3 \mathrm{G}$ will result in significant improvements in spectral efficiency that will allow mobile operators to share their licensed spectrum much more intensively among a larger number of users. Such operator-mediated spectrum sharing, using spectrum managed by
}

such enhancements are on-going and important, in this paper, we focus on mechanisms for sharing spectrum among users directly and across wireless network infrastructures and access rights regimes.

Several approaches have emerged to improve dynamic access to spectrum for new (and existing) users and service providers. These include license-exempt sharing, cooperative sharing and license trading.

License exempt sharing is possible in certain dedicated "unlicensed" bands such as the ISM 2.4 and $5 \mathrm{GHz}$ bands used by 802.11 WLANs, cordless phones and other types of devices subject to Part 15 rules. License exempt use has also been approved for secondary usage by low power ultrawideband devices and, more recently, for higher power opportunistic secondary use sharing of TV bands [2]. Opportunistic systems allow users to temporarily use spectrum when the primary user is not. Typically, these systems require some kind of sensing technology to find idle spectrum and to vacate spectrum if the primary user begins using it again. Cognitive radios have emerged for this kind of use. In both cases, the license-exempt users have secondary usage access rights, which means that they are constrained to operate in such a way as to avoid interfering with the primary access rights holder. The low power mode of operation is sometimes referred to as a spectrum "underlay" because such secondary use devices are expected to operate below the noise floor of the primary user. The higher power, opportunistic mode of sharing anticipated for the TV band "white spaces," is sometimes referred to as an "overlay" since the secondary user's access rights are overlaid on top of the primary user so as to exploit opportunities when the primary user is not actually utilizing the spectrum. While license-exempt usage - whether in dedicated unlicensed or on a secondary usage basis - provides a valuable opportunity for dynamically sharing spectrum, it does not allow the licenseexempt user to reliably control or predict the level of interference that may occur.

Another approach to sharing is cooperative sharing, where the primary and secondary user agree on terms and conditions of sharing (i.e., what frequencies, what time periods, what prices). This kind of cooperative, contract-based sharing is already seen with mobile virtual network operators (MVNOs),

a single network operator (e.g., spectrum licensed for exclusive-use to the operator), will likely utilize many of the same techniques and technologies that market-based sharing will employ, and this approach may prove to be the dominant business model for managing the more intensive sharing of spectrum. 
who use licensed spectrum through negotiation (see [3] for a further discussion).

Finally, it is possible that secondary markets for licenses may emerge. A sufficiently liquid market would make dynamic spectrum reassignment possible.

Table 1 illustrates a taxonomy for the modes of dynamic spectrum sharing. The term "non-cooperative" in this table means that there is no coordination among users beyond what might be implicit in the medium access control (MAC) protocol. In the secondary sharing case, it means no coordination between the primary and secondary users although there may be coordination between secondary users via a MAC protocol. The "sharing" dimension refers to spectrum rights, with primary sharing being peer-based and secondary sharing hierarchical. That is, in primary sharing, all users have equal status whether they are sharing the same spectrum (as in non-cooperative use) or exchanging usage rights (as in cooperative use). Secondary sharing assumes a primary user (i.e., a license holder) and then addresses different means by which idle spectrum may be used without exchanging de jure usage rights (i.e., licenses).

\begin{tabular}{|l|l|l|}
\hline & Non-cooperative & Cooperative \\
\hline Primary sharing & $\begin{array}{l}\text { Unlicensed (eg. } \\
\text { WiFi, Bluetooth })\end{array}$ & $\begin{array}{l}\text { Secondary } \\
\text { spectrum markets }\end{array}$ \\
\hline $\begin{array}{l}\text { Secondary } \\
\text { sharing }\end{array}$ & $\begin{array}{l}\text { Easements, } \\
\text { opportunistic use }\end{array}$ & $\begin{array}{l}\text { Cooperative } \\
\text { sharing }\end{array}$ \\
\hline
\end{tabular}

Table 1 - A taxonomy of dynamic spectrum assignment

Much of the attention in the technical literature has been on non-cooperative approaches to spectrum sharing. Unlicensed use has been an important technical and economic approach to spectrum access that has been highly visible in the product market. Because unlicensed users must share the same RF bands, these systems suffer from unpredictable interference, which may result in a poor or unpredictable quality of service (QoS) or channel capacity. While this is acceptable to some users for some applications, it will clearly not meet all communications needs. As a result, we see calls for unlicensed use as a more general strategy for spectrum management [4], which basically increases the spectrum available for dedicated unlicensed use. This has the effect of spreading existing users over more bands (i.e., increasing SINR) and increasing the (aggregate) channel bandwidth.

While some spectrum managers may be sympathetic to the idea of increasing the allocation for dedicated unlicensed usage, such a reassignment and/or reallocation presents significant political, economic, and regulatory challenges. ${ }^{3}$ The emergence of cognitive radios [5] (CRs) gave rise to the possibility of non-cooperative secondary sharing, which offers

\footnotetext{
${ }^{3}$ Reallocation/reassignment of spectrum access rights confronts many challenges. First, incumbent users/uses must be relocated. Second, because spectrum access rights are scarce, there are multiple stakeholders seeking to secure rights and they often disagree on the best regime for newly reallocated spectrum (e.g., exclusive licensed or unlicensed, license terms, auctions, etc.). Third, selecting a protocol and rules for unlicensed usage in a new allocation (e.g., will it be preemptible? How will it be shared? Etc.) is also likely to be contentious. Overcoming these obstacles is a multidisciplinary challenge.
}

an alternative way to expand unlicensed usage [6]. Some aspects of these systems are described elsewhere in this special issue.

Cooperative spectrum sharing is rooted in the seminal ideas of the Nobel laureate economist Ronald Coase, who argued that scarcity, by itself, does not call for government regulation [7]. He went on to argue that the problem was a lack of clear property rights in spectrum. In a property rights regime, he argued, efficient allocation and assignment of spectrum could be achieved through the use of price mechanisms. While Weiser and Hatfield [8] have argued that property rights in spectrum pose challenges that are not found in tangible goods, the notion that approaches to spectrum assignment that are based on economics has taken hold in spectrum management authorities around the world. In such a regime, access to spectrum would be purchased at a price that should (theoretically) maximize social benefits. Coase's original analysis does not discriminate between primary and secondary sharing.

The spectrum auctions that are used in many countries for the initial assignment of spectrum are an attempt to implement part of this scheme: those users for whom spectrum is most valuable will be willing to pay the most for it. Moreover, those organizations that purchase spectrum would have strong incentives to make economically efficient tradeoffs between spectrum and technology (i.e., modulation, antennas, etc.). The regulator cedes to the market the assignment of the spectrum, and if the license terms are sufficiently flexible (e.g., allow the licensee to choose what the spectrum is to be used for), also part of the spectrum allocation decision. Because regulatory authorities are likely to be less informed than the network operators and users and because the regulatory process imposes significant costs, assignment by auction is preferred by most economists. ${ }^{4}$

Price-based access to spectrum, however, is not without concerns. Critics have noted, for example, that:

- Public service entities (eg., police, fire, ambulance) are poorly positioned to compete effectively for spectrum with commercial entities even though their services may be socially very valuable.

- Valuable new services may not emerge because startup firms rarely have the resources to compete for the spectrum licenses they need.

- Price-based spectrum can favor incumbents over new entrants because of their (i.e., the incumbents') superior capitalization

Spectrum managers have developed a variety of approaches to address these concerns, including "set asides" for public

\footnotetext{
${ }^{4}$ Regulatory processes impose direct and indirect costs. There are the direct costs associated with participation and delay, as well as the indirect costs associated with a potential loss of efficiency. The latter costs may arise as a consequence of regulatory capture (i.e., the process may be high jacked by private interests rather than focused on maximizing social welfare) or bureaucratic impediments (e.g., asymmetric information, red tape) that distort market behavior and lead to less efficient outcomes. Indeed, a major benefit of moving to an auction process (as opposed to assignment by "beauty pagents" is that it reduces the risk of regulatory capture and may serve to break to logjam of trying to reach a timely regulatory settlement.
} 
service entities and small firms. To create the opportunity for firms to innovate, regulators have set aside spectrum for which users do not require licenses and have provided "experimental" licenses in a number of cases.

While spectrum auctions offer a valuable way to transition from a more cumbersome legacy regulatory regime toward more market-based mechanisms, auctions only solve the first assignment problem. To maintain economically optimal spectrum consumption, users need to be able to adjust their spectrum holdings based on changes in technologies and markets over time. In addition, efficient tradeoffs between spectrum and technology can only be made if the current valuations of spectrum and technology investments are known. One obvious way of providing this capability is to have a secondary market for spectrum usage rights (e.g. licenses) in which spectrum licenses that have already been assigned might be traded in real (or near real) time. ${ }^{5}$ Effective secondary markets require a predictable legal framework as well as sufficient liquidity on both the supply and demand sides of the market [9].

In the absence of real spectrum markets (i.e., cooperative primary sharing), secondary spectrum sharing rises as a potential alternative. In this form of DSA, license holders contract with secondary users. Cooperative secondary sharing offers several advantages over non-cooperative DSA, including:

- More spectrum may become available since primary and secondary users could negotiate efficient financial payments to allocate the net benefits of enhanced spectrum sharing; ${ }^{6}$

- These systems could use simpler (less expensive) radios; ${ }^{7}$ and,

- More sophisticated sharing arrangements could be negotiated. ${ }^{8}$

The purpose of this paper is to discuss cooperative approaches to DSA and to report on the results of research on these kinds of systems. The paper will begin by describing some of the tradeoffs inherent in different approaches to spectrum management. This is not intended to be a detailed literature review or a tutorial but rather to situate the

\footnotetext{
${ }^{5}$ The time scale of spectrum trading turns out to be a quite important aspect of an effective trading regime. For example, licensed access rights might be sub-leased or exchanged for durations of months or years; or, for much shorter periods of time measured in seconds to hours. Enabling realtime trading presents additional technical and mechanism challenges associated with keeping the transaction costs of frequent trading manageable that are avoided if access rights are assigned for longer duration. However, a lack of real-time trading may attenuate the expected benefits of secondary spectrum trading.

${ }^{6}$ Primary and secondary users could negotiate "Coasian" bargains to allocate the costs (e.g., increased risk of interference for primary users v. increased costs for interference avoidance by secondary users) and benefits (e.g., capture of otherwise fallow spectrum for expanded usage) of more intensive sharing.

${ }^{7}$ The primary and secondary users could mutually agree on an optimal interference avoidance strategy. This would likely allow secondary use radios to avoid implementing complex sensing and interference avoidance technologies.

${ }^{8}$ The full flexibility of negotiated contracts could be employed to allow novel technical and business models for sharing spectrum access over time, space, and users.
}

discussion of dynamic spectrum access in a larger context. Then, the attributes of opportunistic use will be described so that the contrast with market-based secondary use is clearer. The general approach to secondary use will then be described along with some recent modeling research results. The paper will then describe another approach to DSA based on permanent license exchanges, or spectrum trading, along with some research results. The paper will conclude with a summary and discussion of future directions.

\section{TRADEOFFS IN SPECTRUM MANAGEMENT}

Spectrum management is a complex function that is typically performed by national governments. Spectrum managers must ensure orderly access to the spectrum so that the largest number of high value users/usages may share the $\mathrm{RF}$ frequencies with a minimum of disruptive interference. In addition to solving this technical challenge, spectrum managers seek ways to balance private rights (that is, the rights of the license holders) against public access (that is, the ability of new entrants to use spectrum). This particular tradeoff is seen in the licensed vs. unlicensed debate.

More liberalized spectrum management regimes also seek to balance the ability to take private action (that is, allow licensees to use spectrum for their greatest economic advantage) against social goals or public rights. Social goals and public rights may include such things as ensuring access to diverse points of view to exist in functional democratic societies or competitive market structures. Social goals can place limits on private action (e.g., license rules may limit the re-use of spectrum allocated to broadcasting for mobile communications).

In its effort to balance the interests of different users/uses, spectrum managers must recognize that quality of service requirements are heterogeneous. Some users may have a very low tolerance for in-band interference (e.g., radio telescopes, 3G mobile operators), ${ }^{9}$ while others may be much more tolerant. ${ }^{10}$ Some users may have regular and predictable needs for spectrum access (e.g., 3G mobile operators), while others may have less-predictable needs (e.g., public safety). ${ }^{11}$ These divergent QoS requirements give rise to needs for both predictable vs. unpredictable QoS spectrum rights.

Figure 1 qualitatively relates the range of choices (on a continuum from exclusive use to commons), the attributes (described above) of the extremes of this continuum and the approaches that are being used or proposed. The leftmost side

\footnotetext{
${ }^{9}$ Radio-telescopes are trying to extract information from a very low power signal so any additional interference (even low power) may be disruptive. 3G mobile operators already use their spectrum much more intensively in dense locations to support high usage and so have a lower tolerance for additional (unmanaged) sources of interference.

${ }^{10}$ For example, Wi-Fi radios used for best-effort Internet access scale the data rate to gracefully accommodate congestion. Ultrawideband devices spread the signal over a wide range of frequencies, making them less vulnerable to interference in any particular frequency band.

${ }^{11}$ Public safety may have high peak demands at unpredictable locations and times; whereas mobile operators (partially as a consequence of how they choose to manage their service offerings) may have more regular and predictable end-user demands - although with the movement to mobile broadband, per-user usage is becoming more bursty and less predictable.
} 
of the bottom of the figure represents approaches that use licenses. Each approach is a way of acquiring an exclusive license to use spectrum, from an administrative approach (command and control) to economic ones (auctions and spectrum trading). On the right bottom side are the commons approaches that involve unlicensed approaches. The middle bottom of this figure represent hybrid approaches, such as opportunistic use, market based secondary use, mobile virtual network operators (MVNOs), etc.

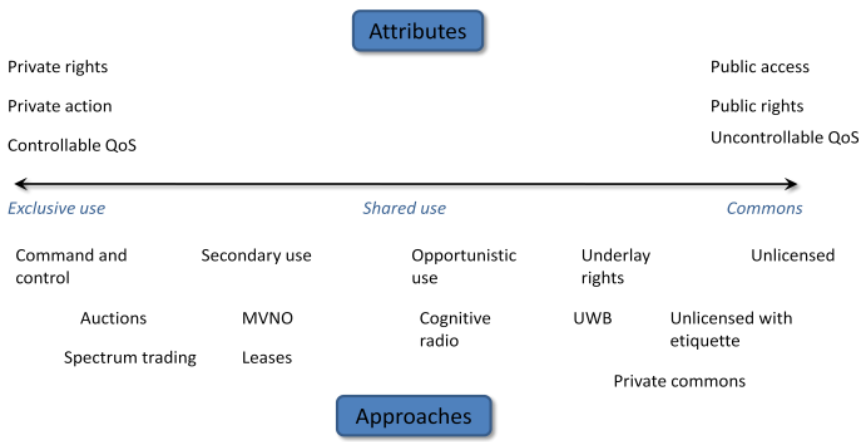

Figure 1 -- Spectrum management attributes and technological approaches

What Figure 1 should make clear is that the choice in regimes is a continuum rather than a simple dichotomy between property rights on the one hand and commons spectrum on the other. Both extremes and everything in between have a balance of rules (regulatory) and market-based forces that contribute to how spectrum is managed. There is no single regime that is best for all situations. Different technologies, business models, and market contexts may call for different optimal modes of spectrum management, and we may expect to see inter-modal competition prospering simultaneously over multiple regimes in markets for similar services. $^{12}$

\section{NON-COOPERATIVE SECONDARY SHARING}

The approach to spectrum sharing that has dominated the technical academic literature has been focused on secondary use. This approach requires the use of cognitive radios [5] that are capable of detecting spectrum holes, matching them to the needs of applications and vacating the spectrum when the primary user begins using it again. Akyildiz et.al. provide a summary of these radios and their research issues [6].

\section{A. Challenges with general cognitive radio systems}

To respond to their environment, cognitive radios must sense their environment to identify spectrum holes, match the spectrum properties to the requirements of the application,

\footnotetext{
${ }^{12}$ Mobile telephony already provides a case in point. We see multiple facilities-based operators offering mobile telephone services using a variety of wireless technologies (GSM, CDMA, WiMAX) and business models (MVNOs, WiFi, traditional service providers), and in competition with other technology platforms (wired telephony, over-the-top VoIP). These services are provided over a diverse range of frequency bands, managed according to the full range of spectrum management regimes (command \& control to fully flexible licensed to unlicensed spectrum, according to management regimes that vary across national sovereign borders).
}

tune the radio to this band and continue sensing to determine if the primary user is beginning to transmit again. In the most general case, this sensing problem is challenging because:

- The sensing has to be performed over the entire band that the radio can be tuned with selectivity equal to the minimum usable channel bandwidth.

- If the primary user's signal is unknown, then more effective matched-filter detection techniques cannot be used.

- Sensing most probably needs to be cooperative to overcome the hidden node problem and to define the geographic area covered by a potential spectrum hole.

- Detection has to be rapid, especially when a radio is using a band, because it must be vacated with little delay when a primary user restarts transmission.

In addition to sensing challenges, cognitive radios need a MAC protocol to coordinate their use of spectrum holes. Ongoing sensing can only be done when the radios of opportunistic users are off, which means that the spectrum hole cannot be used with a $100 \%$ duty cycle.

Combining the inefficiencies due to sensing and MACbased contention resolution invites researchers to consider alternatives to general opportunistic systems. These alternatives include narrowing the scope of the cognitive radio problem and considering non-opportunistic approaches.

\section{B. Defining spectrum holes}

In most cases, the concept of a spectrum hole (i.e., unused spectrum in the time, frequency and geospatial dimension) is thought of from an FDMA perspective. That is, the systems assume that some frequency bandwidth is available for a certain time (that is much greater than the time it takes to find, evaluate and switch to it) in a certain geographic area. Many modern communications systems do not use FDMA; rather they use OFDMA (LTE, WiMAX), a variant of TDMA (such as GSM) or CDMA (UMTS, CDMA 2000). In these systems, defining a "spectrum hole" is more difficult and requires coordination between the primary and secondary user [10].

The degree of coordination depends on the multiple access scheme used by the primary user. In OFDMA systems, detecting a spectrum hole is much more likely if the subcarriers of the primary user are known, which requires some coordination between primary and secondary users (albeit not on a real-time or operational level). Exploiting TDMA spectrum holes requires time synchronization between the primary and secondary user. Finally, CDMA systems require code coordination in addition to time synchronization. Without this level of coordination, detecting unused (or underused) spectrum is difficult.

\section{The special case of television white spaces}

In [2], the US Federal Communications Commission approved the operation of cognitive radios in the television "white spaces". Since the location, power levels, channel bandwidths and signal strengths of television transmitters are known, the white space detection problem is simplified considerably. The white spaces are used by wireless microphones, so sensing is still necessary. By permitted 
unlicensed operation in white spaces, the FCC opted for a blend between non-cooperative primary use and noncooperative secondary use. It is a blend between these because the secondary use is from an allocation perspective but not an assignment perspective in that the white space devices (WSDs) do not use otherwise licensed spectrum. Thus, the WSDs appear as primary users of unassigned spectrum that has been allocated to television service but has either not been assigned or is not being used.

In any case, WSDs must have a MAC protocol (e.g., [11]) to share the spectrum among competing users. They may also need to engage in cooperative sensing, meaning that there may need to be a communication channel among them.

\section{Implications for efficient spectrum use}

Non-cooperative secondary sharing beyond FDMA systems requires a degree of coordination that is unlikely to be found in opportunistic systems that are based on the current models of sensing. Thus, spectrum that might otherwise be open to sharing would remain undetected by cognitive radios and thus remain unused. Cooperative approaches make spectrum that is technologically "hidden" open for sharing.

\section{COOPERATIVE SECONDARY SHARING}

Non-cooperative secondary sharing allows for the emergence of a relatively decentralized access system. This approach requires either a cognitive radio or one that has a "white spaces" database that determines its operating parameters (see, for example [12]). For the foreseeable future, cognitive radios will be costly and will consume considerable energy, making mobility challenging, especially for handsets. Sensing-based systems will almost certainly require cooperative sensing to overcome the hidden terminal problem and to establish the geographic perimeter of spectrum holes, which adds to radio overhead. Finally, non-cooperative secondary sharing requires a MAC protocol to mediate among competing secondary users, which also imposes overhead.

Many of these problems can be ameliorated if secondary usage is cooperative. If the primary user is directly involved in the secondary use decision, then there is no longer a unilateral need for sensing or for a MAC protocol for the secondary use radios. This makes it possible to design secondary use radios that are less costly and more energy efficient. Spectrum that might be "hidden" in TDMA, CDMA or OFDMA systems can be made available because the closer coordination that is required can be negotiated. The primary and secondary users can negotiate over the optimal allocation of interference avoidance costs and benefits from increased sharing. Finally, the primary user may be willing to open spectrum that they might otherwise seek to protect (e.g., by sending null signals during idle periods) because:

- The primary user can exercise control over the QoS they offer to their users, and,

- The primary user can negotiate financial incentives to open spectrum to secondary users.

Given these advantages, why is there less attention being paid to cooperative secondary sharing than to non-cooperative sharing?

\section{A. Research Questions}

The research literature on cooperative secondary sharing is relatively sparse but is growing. Some research questions that arise include:

- What is the structure of the incentives for primary users (license-holders) to share spectrum with (potential) secondary users?

- Under what circumstances would users choose secondary use from their array of communications options? How is this affected by the particular frequency band, geographical constraints and access technology?

- Are cooperative secondary use approaches more efficient (technically, economically, and/or politically) than noncooperative approaches?

- What are the implications of different approaches to negotiating cooperative agreements (eg., markets, bilateral negotiations, etc.)?

- What are the implications of different access technologies (eg. FDMA, TDMA, CDMA, OFDM) on secondary sharing behavior?

- What kinds of strategic behaviors might we expect among primary and secondary usage, and how might the deleterious behaviors be ameliorated?

- What users, applications and business models are most likely to find cooperative sharing attractive?

- What other changes are needed in radio design to make flexible spectrum sharing contracts easier to negotiate (e.g., standardization?) and enforce?

- What changes in regulatory policy are needed to foster and facilitate efficient cooperative sharing? What concerns may cooperative sharing raise for antitrust or asymmetric bargaining power?

\section{B. Research Results}

There is a growing literature seeking to model DSA secondary use sharing regimes and the potential benefits its promises. Weiss [3] pointed out that some kinds of cooperative secondary use (Type I) already exist in practice in the form of MVNO agreements, which are the result of bilateral negotiations. This suggests (1) that incentives for primary and secondary users exist and (2) that different types of sharing might result in different negotiating mechanisms.In [13], Chapin and Lehr provide a high level view on approaches to sharing and in [14], they suggest changes in radio design to promote access by new entrants. In [15] Lehr and Jesuale discuss an approach to moving toward cooperative sharing.

Peha and Panichpapiboon [16] considered the incentives that a primary user (license holder) would have in sharing spectrum to secondary users. They showed that, for GSM operators, strong incentives to allow secondary use exist since license holders can monetize otherwise idle spectrum.

Grandblaise et.al. [17] report on the results of the $E^{2} R$ research project. They consider spectrum sharing between system operators as well as between operators and end users. They build a model based on recurrent auctions to determine the efficiency gain of a DSA system assuming profit- 
maximizing operators/users. Potential users bid for spectrum usage rights based on their projected needs (and revenues) for the next period. Grandblaise et al. show that different spectrum assignment approaches apply at different combinations of traffic load and per-cell blocking probabilities. DSA approaches are most effective for low to moderate load levels whilst fixed spectrum assignment is more effective at higher load levels.

Tonmukayakul and Weiss [18] studied the behavior of potential secondary users in a market-based negotiating system using agent-based computational economics. In their model, users could choose between acquiring a license, purchasing secondary use rights on a market or using unlicensed spectrum. Their model included QoS factors as well as the possibility of opportunistic behavior for both primary and secondary users. Figure 2 is an example of the results of this model.

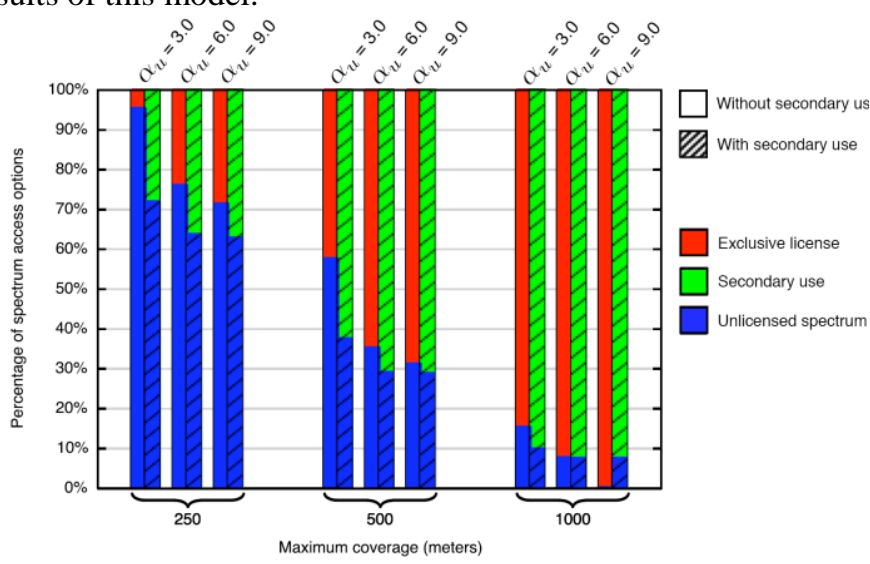

Figure 2 -- Secondary use in a synthetic market

In this figure (taken from [19]), each of the clusters of bars represent cell sizes, and the pairs of bars in each cluster represent user sensitivity to interference. The left side of each pair of bars (not cross-hatched) represents the condition where secondary use does not exist and the right, cross-hatched section describes user choices with secondary use. So, for example, if we take a $500 \mathrm{~m}$ cell with $\alpha_{u}=3.0$, the simulation results indicate that approximately $60 \%$ of potential secondary users choose unlicensed if no secondary use exists, while this number becomes approximately $40 \%$ if secondary use exists. The demand for licenses among secondary users disappears in all scenarios in the presence of secondary use under this set of modeling parameters ${ }^{13}$. Note that users in environments characterized by small cells are more likely to choose unlicensed use because the interference is lower.

Al Daoud et al. [20] studied pricing in cooperative secondary sharing without particular attention to the technical coordination requirements of CDMA. The main objective in selecting CDMA was to focus on pricing without requiring the total absence of interference. Thus, they are able to define prices based on the induced interference. They show that profits are improved when interference-based pricing is introduced when compared with a simpler, flat pricing scheme.

\footnotetext{
${ }^{13}$ Note that demand for licenses would still exist for primary users, though this is not in the scope of the model
}

\section{COOPERATIVE PRIMARY SHARING}

DSA need not be limited to temporary transfers of rights as is the case in cooperative secondary sharing. If markets are sufficiently frictionless, then a scenario that is equally viable is one in which spectrum usage rights are purchased as needed and sold when they are no longer needed. That is, a secondary market for spectrum licenses. ${ }^{14}$

Spectrum trading has been discussed in the academic literature for some time. The tradeoffs involved have been summarized by the OECD [21]. Spectrum trading offers some advantages over both dedicated unlicensed or secondary use. These include:

- There is no need to negotiate geographical, temporal, spectral or air interface parameters since licenses are transferred;

- Interference is subject to regular interference regulations;

- No negotiation protocol need be established; and,

- Software radios are technologically adequate (i.e., the added complexity of cognitive radio-driven sensing and active avoidance is not required).

But there are numerous challenges that need to be addressed as well, including:

- Will spectrum markets be sufficiently liquid and frictionless on both the supply and demand side?

- Since spectrum at different frequencies (and locations/times) is not fungible, how can a tradable commodity be constructed?

- How can inefficient fragmentation of a spectrum band be avoided?

- How should a market be administered? Should the government or a third-party spectrum broker be in charge?

- What regulatory interventions may be required? What are the cost/consequences of those interventions?

- What are the tradeoffs involved in the trading architectures chosen (eg. Broker vs. market vs. auction)?

Although often discussed, additional research is needed to understand how such a secondary market to enable primary sharing might operate in practice. Caiecedo and Weiss [22] have constructed a model using agent-based computational economics. They focused on studying the boundaries of viability in spectrum trading markets. They found that, given their modeling parameters, that a minimum of six active market participants were necessary, and that the amount of spectrum available for trading had to be calibrated to the traffic that this spectrum needed to carry (albeit with an error tolerance of $+/-50 \%$ ).

Yoon [23] performed a comparative study of different trading mechanisms (i.e., broker-based, auctions and direct trading). Her study found that provider's profits were superior under auctions while the subscriber's surplus was constant across all trading mechanisms. However, when considering

\footnotetext{
${ }^{14}$ The market is a secondary market in the same sense that the stock exchange is a secondary market. In equities, the primary market is the Initial Public Offering (IPO) whereas in spectrum the primary market would be an auction or beauty contest
} 
the dynamic trading behavior under this mechanism, the research found that a large incumbent could monopolize spectrum in the absence of regulation. Using spectrum caps can address concentration, but this work does not address the possible deadweight losses associated with this intervention. Yoon's model suggests that direct trading provides a more active market with or without regulation. Finally, she considers market scenarios and business models related to secondary use and secondary markets.

\section{SUMMARY AND FUTURE WORK}

Much of the attention in the technical literature has been on improving the functions of cognitive radios for noncooperative secondary sharing. The purpose of this paper is to:

- situate that work in the broader context of dynamic spectrum access,

- introduce readers to research in the cooperative sharing domain, and,

- $\quad$ point out future research that would be valuable in this cooperative sharing domain.

This research requires substantial knowledge of both the underlying technology models as well as economic and business models.

\section{ACKNOWLEDGMENT}

The authors would like to acknowledge the research efforts of Dr. Arnon Tonmukayakul and Dr. Carlos Caicedo. Their research has contributed to many of the ideas in this paper.

\section{REFERENCES}

[1] Mark McHenry, "NSF Spectrum Occupancy Measurements Project Summary," National Science Foundation, Arlington VA, NSF Award Number ANI-0335272, 2005.

[2] Federal Communications Commission, In the Matter of Unlicensed Operation in the TV Broadcast Bands, Second Report and Order and Memorandum Opinion and Order, November 14, 2008, FCC 08-260.

[3] Martin BH Weiss, "Secondary use of spectrum: a survey of the issues," Info, vol. 8, no. 2, pp. 74-82, 2006.

[4] William Lehr, "The Economic Case for Dedicated Unlicensed Spectrum Below 3GHz," New America Foundation, Washington DC, Spectrum Series Working Paper 9, 2004.

[5] Joseph Mitola, Cognitive Radio, 1998, Licentiate proposal, KTH, Stockholm, Sweden.

[6] Ian F Akyilidiz, Won-Yoel Lee, Mehmet C. Vuran, and Shantidev Mohanty, "NeXT generation/dynamic spectrum access/cognitive radio wireless networks: A survey," Computer Networks, vol. 50, pp. 2127-2159, 2006.

[7] Ronald H. Coase, "The Federal Communications Commission," Journal of Law and Economics, vol. 2, pp. 140, October 1959.

[8] Philip J. Weiser and Dale Hatfield, "Spectrum Policy Reform and the Next Frontier of Property Rights," George
Mason Law Review, vol. 15, no. 3, pp. 549-609, 2008.

[9] Carlos E Caicedo and Martin BH Weiss, "On the Viability of Spectrum Trading Markets," in Telecommunications Policy Research Conference, Arlington, VA, 2009.

[10] Arnon Tonmukayakul and Martin BH Weiss, "Secondary Use of Radio Spectrum: A Feasibility Analysis," in Telecommunications Policy Research Conference, Arlington VA, 2004.

[11] Ranveer Chandra, Thomas Moscibroda, Rohan Murty, Matt Welsh Paramvir Bahl, "White Spaces Networking with WiFi like Connectivity," in Proceedings of ACM SIGCOMM, Barcelona, 2009, pp. 27-38.

[12] Google, "Introducing the White Spaces Database Group," Google Public Policy Blog, http://googlepublicpolicy.blogspot.com/2009/02/introducingwhite-spaces-database-group.html, 2009.

[13] J.M. Chapin and W.H. Lehr, "The Path to Market Success for Dynamic Spectrum Access Technology," IEEE Communications Magazine, vol. 45, no. 5, pp. 96-103, May 2007.

[14] J.M. Chapin and Lehr W.H., "Time-limited leases in radio systems," IEEE Communications Magazine, vol. 45, no. 6, pp. 76-82, June 2007.

[15] W.H. Lehr and N. Jesuale, "Public Safety Radios Must Pool Spectrum," IEEE Communications Magazine, vol. 47, no. 3, pp. 103-109, March 2009.

[16] Jon M. Peha and Sooksan Panichpapilboon, "Real time secondary markets for spectrum," Telecommunications Policy, vol. 28, pp. 603-618, 2004.

[17] David Grandblaise et al., "Techno-Economics of Collaborative-based Secondary Spectrum Usage," in IEEE New Frontiers in Dynamic Spectrum Access Networks, Baltimore MD, 2005, pp. 317-327.

[18] Arnon Tonmukayakul and Martin B.H. Weiss, "A study of secondary spectrum use using agent-based compuational economics," Netnomics, vol. 9, pp. 125-151, 2008.

[19] Arnon Tonmukayakul, "An Agent-Based Model for Secondary Use of Radio Spectrum," University of Pittsburgh, Pittsburgh PA, PhD Dissertation 2007.

[20] Ashraf Al Daoud, Murat Alanyali, and David Starobinski, "Secondary pricing of spectrum in cellular CDMA networks," in IEEE International Symposium on New Frontiers in Dynamic Spectrum Access Networks, Dublin Ireland, 2007.

[21] OECD, "Secondary Markets for Spectrum: Policy Issues," Paris, DSTI/ICCP/TISP(2004)11/FINAL, 2005.

[22] Carlos E Caicedo and Martin B.H. Weiss, "On the Viability of Spectrum Trading Markets," in Telecommunications Policy Research Conference, Arlington VA, 2009.

[23] Hyen-Young Yoon, "Spectrum Management Policy Studies for Next Generation Wireless Industry: A Techno-Economic Approach," Seoul National University, College of Engineering, Seoul, Korea, PhD Dissertation 2009. 
Martin B.H. Weiss (M'76-SM'81-F'87) and the other authors may include biographies at the end of regular papers. Biographies are often not included in conference-related papers. This author became a Member (M) of IEEE in 1976, a Senior Member (SM) in 1981, and a Fellow (F) in 1987. The first paragraph may contain a place and/or date of birth (list place, then date). Next, the author's educational background is listed. The degrees should be listed with type of degree in what field, which institution, city, state, and country, and year degree was earned. The author's major field of study should be lower-cased.

The second paragraph uses the pronoun of the person (he or she) and not the author's last name. It lists military and work experience, including summer and fellowship jobs. Job titles are capitalized. The current job must have a location; previous positions may be listed without one. Information concerning previous publications may be included. Try not to list more than three books or published articles. The format for listing publishers of a book within the biography is: title of book (city, state: publisher name, year) similar to a reference. Current and previous research interests end the paragraph.

The third paragraph begins with the author's title and last name (e.g., Dr. Smith, Prof. Jones, Mr. Kajor, Ms. Hunter). List any memberships in professional societies other than the IEEE. Finally, list any awards and work for IEEE committees and publications. If a photograph is provided, the biography will be indented around it. The photograph is placed at the top left of the biography. Personal hobbies will be deleted from the biography.

William H. Lehr 